Proceedings of the Edinburgh Mathematical Society (2002) 45, 513-522 (C)

DOI:10.1017/S0013091501001043 Printed in the United Kingdom

\title{
GROUPS WITH THE MAXIMAL CONDITION ON NON-BFC SUBGROUPS. II
}

\author{
MARTYN R. DIXON AND LEONID A. KURDACHENKO \\ ${ }^{1}$ Department of Mathematics, University of Alabama, Tuscaloosa, \\ AL 35487-0350, USA (mdixon@gp.as.ua.edu) \\ ${ }^{2}$ Department of Algebra, University of Dnepropetrovsk, Vulycya Naukova 13, \\ Dnepropetrovsk 50, 49050, Ukraine (mmf@ff.dsu.dp.ua)
}

(Received 14 November 2001)

Dedicated to to the memory of D. I. Zaicev on the 60th anniversary of his birth.

\begin{abstract}
A group $G$ is called a group with boundedly finite conjugacy classes (or a BFC-group) if $G$ is finite-by-abelian. A group $G$ satisfies the maximal condition on non-BFC-subgroups if every ascending chain of non-BFC-subgroups terminates in finitely many steps. In this paper the authors obtain the structure of finitely generated soluble-by-finite groups with the maximal condition on nonBFC subgroups.
\end{abstract}

Keywords: BFC-group; maximal condition; soluble-by-finite

AMS 2000 Mathematics subject classification: Primary 20E15

Secondary 20F16; 20F24

\section{Introduction}

A group $G$ is called a group with boundedly finite conjugacy classes (or a BFC-group for short) if there is an integer $m$ such that $\left|G: C_{G}(g)\right| \leqslant m$, for all $g \in G$, or, equivalently, by a theorem of Neumann (see [11, Theorem 4.35]) if $G$ is finite-by-abelian. In a previous paper [5] we discussed the class of groups with the maximal condition on non-BFCsubgroups. We recall from [5] that a group $G$ satisfies max-(non-BFC) if every ascending chain of non-BFC-subgroups terminates in finitely many steps. The condition max-(nonBFC) is, of course, dual to the condition min-(non-BFC) discussed by Bruno and Phillips in [1], and our paper [5] and the current paper represent a further contribution to the theory of groups with certain chain conditions on their subgroups (see, for example, [2$\mathbf{4}, \mathbf{7}, \mathbf{8}, \mathbf{1 0}]$ to name but a few articles concerned with this sort of topic).

It is quite easy to see that if a group $G$ satisfies max-(non-BFC), then $G$ is either finitely generated or is a locally FC-group (i.e. every finitely generated subgroup has all conjugacy classes finite), and in [5] we obtained the structure of locally FC-groups with max-(non-BFC). In this paper we take up the case of finitely generated groups with max-(non-BFC). Here we consider the case of finitely generated soluble-by-finite groups. 
Together with the results of $[\mathbf{5}]$ the results we obtain here enable us to give the structure of all locally soluble-by-finite groups with max-(non-BFC). This is just about as far as we can go with our current techniques. One obstacle to further progress (for example in trying to obtain a classification theorem for locally graded groups) is the possibility that there exists an infinite finitely generated residually finite $p$-group, for some prime $p$, with all subgroups either finite or of finite index. Such a group (if it exists) necessarily satisfies max-(non-BFC).

In $\S 2$ of this paper we present some further results on modules, analogous to those in $\S 5$ of [5]. In $\S 3$ we obtain our main results, Theorems 3.2 and 3.4. We state Theorem 3.2 here to indicate the flavour of the kind of result we obtain.

Theorem 3.2. Let $G$ be a finitely generated soluble-by-finite group satisfying max(non-BFC). Suppose that $S$ is the soluble radical of $G$ and that $K$ is that term of the derived series of $S$ such that $S / K$ is finitely generated but $K / K^{\prime}$ is not finitely generated, and suppose that $K$ is a BFC-group. Then $G$ has a series of normal subgroups $F \leqslant A \leqslant G$ satisfying the following conditions:

(i) $F$ is finite;

(ii) $A / F$ is abelian;

(iii) $G / A$ is finitely generated, abelian-by-finite and torsion-free; and

(iv) $A / F$ is a finitely generated $\mathbb{Z}\langle g\rangle$-module for each element $g \in G \backslash A$.

Furthermore, if $K / K^{\prime} K^{p}$ is finite for each prime $p$, then $F$ may be chosen such that $A / F$ is torsion-free and minimax. In this case $G$ is also minimax.

Naturally this paper has a heavy dependence on [5]. Our notation and terminology follow that paper where they are not explained here.

\section{Some results on modules}

We refer the reader to $[\mathbf{5}, \S 5]$ for preliminary information concerning modules over the integral group ring of an infinite cyclic group. We will be concerned with the action of an element of infinite order on bounded abelian groups.

Lemma 2.1. Let $G$ be a group satisfying max-(non-BFC). Suppose that $g$ is an element of infinite order and $A$ is a $\langle g\rangle$-invariant infinite abelian bounded $p$-subgroup, for some prime $p$. If $A$ is a periodic $\mathbb{Z}\langle g\rangle$-module, then $\langle A, g\rangle$ is a BFC-group and, in particular, $C_{\langle g\rangle}(A) \neq 1$.

Proof. First we suppose that $A$ is an elementary abelian $p$-group. Let $J=F_{p}\langle g\rangle$. Since $A$ is $J$-periodic, $A=\bigoplus_{P \in \Pi_{J}(A)} A_{P}$, where $A_{P}$ is the $P$-component of $A$. Since every $A_{P}$ is $\langle g\rangle$-invariant, [5, Lemma 2.2] implies that we may assume that the set $\Pi_{J}(A)$ is finite. Let $B_{P}$ be a basic submodule of $A_{P}$. Lemma 5.1 of [5] shows that $A_{P}=B_{P}$ and hence $A_{P}=\bigoplus_{\lambda \in \Lambda} C_{\lambda}$, where $C_{\lambda}=c_{\lambda} J$ is a cyclic $J$-submodule for each $\lambda \in \Lambda$. If 
$P \neq(g-1) J$, then $\left[\mathbf{5}\right.$, Lemma 2.2] implies that $\Lambda$ is finite and hence $A_{P}$ is finite, since $C_{\lambda} \cong_{J} J / \operatorname{Ann}_{J}\left(c_{\lambda}\right)$, for each $\lambda \in \Lambda$. Hence $A=B \oplus C$, where $B$ is a finite $J$-submodule, and $C=C_{A}(g)=A_{Q}$, where $Q=(g-1) J$. Thus $(A\langle g\rangle)^{\prime} \leqslant B$ so $H=A\langle g\rangle$ is a BFC-group.

In the general case, since $A$ is a bounded infinite abelian $p$-group $E=A / A^{p}$ is infinite and since $A$ is $\mathbb{Z}\langle g\rangle$-periodic it follows that $E$ is $F_{p}\langle g\rangle$-periodic. By the above, $E$ contains a finite $\langle g\rangle$-invariant subgroup $U$ such that $\langle E, g\rangle / U$ is abelian. Hence $\langle A, g\rangle$ contains a normal subgroup $V$ such that $\langle A, g\rangle / V$ is an infinite elementary abelian $p$ group. Lemma 2.3 of [5] implies that $H=\langle A, g\rangle$ is a BFC-group. Thus, for each $a \in A$, $\left|H: C_{H}(a)\right|$ is bounded and hence $g^{n} \in C_{H}(A)$, for some integer $n$, as required.

Lemma 2.2. Let $G$ be a group satisfying max-(non-BFC). Suppose that $g$ is an element of infinite order and $A$ is a $\langle g\rangle$-invariant infinite bounded abelian subgroup. If $\langle A, g\rangle^{\prime}$ is infinite, then $A$ is a finitely generated $\mathbb{Z}\langle g\rangle$-module.

Proof. First we suppose that $A$ is an elementary abelian $p$-group, for some prime $p$. Let $J=F_{p}\langle g\rangle$ and let $T$ be the $J$-periodic part of $A$. Lemma 2.1 implies that $T \neq$ $A$. Let $M=\left\{c_{\lambda} \mid \lambda \in \Lambda\right\}$ be a maximal $J$-independent set of elements in $A$. Then $C=M J=\bigoplus_{\lambda \in \Lambda} c_{\lambda} J$ is a free $J$-submodule. Since $\operatorname{Ann}_{J}\left(c_{\lambda}\right)=0$, we have $\left\langle c_{\lambda}, g\right\rangle \cong$ $\left\langle c_{\lambda}\right\rangle ح\langle g\rangle$, and hence $\left\langle c_{\lambda}, g\right\rangle^{\prime}$ is infinite for each $\lambda \in \Lambda$. Since $c_{\lambda} J$ is $\langle g\rangle$-invariant, [5, Lemma 2.6] implies that $\Lambda$ is finite so that $C$ is a finitely generated $J$-module. Clearly, $\langle C, g\rangle^{\prime}$ is also infinite so [5, Lemma 2.6] shows that $A / C$ satisfies max- $F_{p}\langle g\rangle$. In particular, $A / C$ is a finitely generated $F_{p}\langle g\rangle$-module and hence so is $A$.

Next, suppose that $A$ is a bounded $p$-group, for some prime $p$. Let $A_{i}=\Omega_{i}(A)$, for $i \in \mathbb{N}$. There is an integer $k$ such that $A_{k}=A$. For $a \in A_{i}$ the map $\phi_{i}: a \mapsto a^{p^{i-1}}$ is a $\mathbb{Z}\langle g\rangle$-endomorphism from $A_{i}$ to $A_{1}$, so that $A_{i} / A_{i-1}=A_{i} / \operatorname{Ker} \phi_{i} \cong \operatorname{Im} \phi_{i} \leqslant A_{1}$. If $\left\langle A_{1}, g\right\rangle$ is a BFC-group, then $A_{1}$ is $J$-periodic and hence $A_{i} / A_{i-1}$ is $J$-periodic for $1 \leqslant i \leqslant k$. It follows that $A$ is $\mathbb{Z}\langle g\rangle$-periodic, and Lemma 2.1 implies that $\langle A, g\rangle$ is a BFC-group. This contradiction shows that $\left\langle A_{1}, g\right\rangle^{\prime}$ is infinite and hence $A_{1}$ is a finitely generated $J$-module. Thus $A_{i} / A_{i-1}$ is a finitely generated $J$-module for $1 \leqslant i \leqslant k$ and $A$ is a finitely generated $\mathbb{Z}\langle g\rangle$-module, in this case.

Finally, in the general case, let

$$
A=\operatorname{Dr}_{p \in \pi(A)} A_{p},
$$

where $A_{p}$ is a Sylow $p$-subgroup of $A$. If, for every $p \in \pi(A)$, the subgroup $\left\langle A_{p}, g\right\rangle$ is a BFC-group, then the finiteness of $\pi(A)$ implies that $(A\langle g\rangle)^{\prime}$ is finite. Hence there is a prime $p$ such that $\left\langle A_{p}, g\right\rangle$ has infinite derived subgroup. Then $A_{p}$ is a finitely generated $\mathbb{Z}\langle g\rangle$-module, and by [5, Lemma 2.6] $A / A_{p}$ satisfies max- $\mathbb{Z}\langle g\rangle$. Hence $A / A_{p}$ is a finitely generated $\mathbb{Z}\langle g\rangle$-module, and the result follows.

Next we consider the case when $A$ is torsion-free. 
Lemma 2.3. Let $G$ be a group satisfying max-(non-BFC). Suppose that $g$ is an element of infinite order and $A$ is a $\langle g\rangle$-invariant abelian torsion-free subgroup. If $\langle A, g\rangle^{\prime}$ is infinite, then $A$ is a finitely generated $\mathbb{Z}\langle g\rangle$-module.

Proof. First we suppose that $A$ is $\mathbb{Z}\langle g\rangle$-periodic. Since $\langle A, g\rangle^{\prime}$ is infinite there is an element $a_{1} \in A$ such that $g \notin C_{G}\left(a_{1}\right)$. Let $A_{1}=a_{1} \mathbb{Z}\langle g\rangle=\left\langle a_{1}\right\rangle^{\langle g\rangle}$ and $E=A \otimes_{\mathbb{Z}} \mathbb{Q}$. Then $E$ is a $\mathbb{Q}\langle g\rangle$-periodic module and $\mathbb{Q}\langle g\rangle$ is a principal ideal domain. We have $a_{1} \mathbb{Q}\langle g\rangle \cong$ $\mathbb{Q}\langle g\rangle / \operatorname{Ann}_{\mathbb{Q}\langle g\rangle}\left(a_{1}\right)$. Since $\operatorname{Ann}_{\mathbb{Q}\langle g\rangle}\left(a_{1}\right) \neq 0$ it follows that $\operatorname{dim}_{\mathbb{Q}}\left(\mathbb{Q}\langle g\rangle / \operatorname{Ann}_{\mathbb{Q}\langle g\rangle}\left(a_{1}\right)\right)$ is finite. Thus $a_{1} \mathbb{Q}\langle g\rangle$ has finite torsion-free rank, and hence the torsion-free rank of $A_{1}$ is also finite. By a theorem of Hall [11, Corollary 1-Lemma 9.53], $A_{1}$ contains a free abelian subgroup $C$ such that $A_{1} / C$ is periodic and $\pi\left(A_{1} / C\right)$ is finite. Since $A_{1}$ has finite torsion-free rank, $C$ is finitely generated, and hence $A_{1}$ is minimax. If $A_{1}\langle g\rangle$ is a BFCgroup, then it is abelian since $\left(A_{1}\langle g\rangle\right)^{\prime} \leqslant A_{1}$, a torsion-free group. This contradicts the choice of $a_{1}$ and hence $\left(A_{1}\langle g\rangle\right)^{\prime}$ is infinite. Since $\langle g\rangle$ has the maximum condition, $A / A_{1}$ satisfies max- $\langle g\rangle$ and hence $A$ is a finitely generated $\mathbb{Z}\langle g\rangle$-module in this case.

Next let $T$ be the $\mathbb{Z}\langle g\rangle$-periodic part of $A$ and suppose that $T \neq A$. Then the argument of Lemma 2.2 can be used to show that $A$ is a finitely generated $\mathbb{Z}\langle g\rangle$-module.

Finally, we obtain the main result of this section.

Lemma 2.4. Let $G$ be a group satisfying max-(non-BFC). Suppose that $g$ is an element of infinite order and $A$ is a $\langle g\rangle$-invariant abelian subgroup with bounded torsion subgroup. If $\langle A, g\rangle^{\prime}$ is infinite, then $A$ is a finitely generated $\mathbb{Z}\langle g\rangle$-module.

Proof. Let $T$ be torsion subgroup of $A$. Since $T$ is bounded, $A=T \times B$ for some torsion-free subgroup $B$ and there is an integer $k$ such that $T^{k}=1$. Let $E=A^{k}$. Then $E \leqslant B$ and $E$ is a $\langle g\rangle$-invariant torsion-free subgroup of $A$. If $\langle E, g\rangle^{\prime}$ is infinite, then Lemma 2.3 implies that $E$ is a finitely generated $\mathbb{Z}\langle g\rangle$-module. Also $A / E$ satisfies max$\mathbb{Z}\langle g\rangle$, by [5, Lemma 2.6], and hence $A / E$ is a finitely generated $\mathbb{Z}\langle g\rangle$-module and the conclusion holds. Thus suppose that $\langle E, g\rangle$ is a BFC-group. Since $(E\langle g\rangle)^{\prime} \leqslant E$ and $E$ is torsion-free, $E\langle g\rangle$ is abelian and hence $g \in C_{G}(A / T)$. Thus if $T$ is finite then $A\langle g\rangle$ is a BFC-group, contrary to hypothesis. Hence $T$ is infinite. Suppose that $\langle T, g\rangle$ is a BFC-group. Then $T$ contains a $\langle g\rangle$-invariant subgroup $U$ such that $T / U$ is an infinite elementary abelian $p$-group for some prime $p$ and $g \in C_{G}(T / U)$. Again we have $A / U=$ $T / U \times C / U$ for some torsion-free subgroup $C / U$. Let $D / U=(A / U)^{p}$. Then $D / U \leqslant C / U$ and $D / U$ is a $\langle g\rangle$-invariant torsion-free subgroup of $A / U$. Thus $[A / D, g] \leqslant T D / D$ so $\langle A / D, g D\rangle$ is nilpotent. Since $A / D$ is an infinite elementary abelian $p$-group, Lemmas 5.3 and 2.3 of [5] show that $A\langle g\rangle$ is a BFC-group. This contradiction shows that $(T\langle g\rangle)^{\prime}$ is infinite. Since $\langle g\rangle$ has the maximum condition, $A / T$ satisfies max- $Z\langle g\rangle$ and hence $A / T$ is a finitely generated $\mathbb{Z}\langle g\rangle$-module. By Lemma $2.2 T$ is also a finitely generated $\mathbb{Z}\langle g\rangle$-module, whence so is $A$. 


\section{Finitely generated soluble-by-finite groups}

In this section we discuss finitely generated soluble-by-finite groups $G$ with max-(nonBFC) and obtain our main results. We shall use the following notation throughout this section. We shall let $S$ denote the soluble radical of $G$ and let $S=S_{0} \geqslant S_{1} \geqslant \cdots \geqslant S_{n}=1$ be the derived series of $S$. We shall assume that $S$ is not polycyclic, in which case there is a least integer $t$ such that $S / S_{t}$ is polycyclic, but $S_{t} / S_{t+1}$ is not finitely generated. We shall denote $S_{t}$ by $K$. It turns out that the situation is slightly different depending upon whether or not $K$ is a BFC-group. We have found it convenient to separate the following technical lemma off from the main argument.

Lemma 3.1. Let $G$ be a finitely generated soluble-by-finite group satisfying max(non-BFC) and suppose that $\mathcal{S}=\left\{H \triangleleft G \mid K \leqslant H\right.$ and $\left.\left|H^{\prime}\right|<\infty\right\}$. Let $A$ be a maximal element of $\mathcal{S}$ and $F$ a finite $G$-invariant subgroup of $A$ such that $A / F$ is abelian. If $B$ is a $G$-invariant subgroup of $A$ such that $F \leqslant B$ and $|A: B|$ is finite, then

$$
C_{G / F}(B / F)=A / F \text {. }
$$

Proof. First note that since $G / K$ is polycyclic-by-finite, $\mathcal{S}$ has the maximal condition and hence $\mathcal{S}$ has indeed got maximal elements. Note also that $A$ is not finitely generated, otherwise $A$ has the maximal condition, whence $K$ is finitely generated, a contradiction.

Let $C / F=C_{G / F}(B / F)$. Then $C / F$ is central-by-polycyclic-by-finite. Since $A / F$ is not finitely generated neither is $B / F$. If $C / F$ is finitely generated, then $C / F$ has max-n, by a theorem of Hall (see [11, Theorem 5.34]) and the central subgroups of $C / F$ therefore have the maximum condition, which is a contradiction. Hence $C$ is a locally FC-group.

Since $K \leqslant A \leqslant C$ the lemma will follow, using the maximal choice of $A$ once we show that $C$ is a BFC-group. If $A / A^{\prime} A^{p}$ is infinite for some prime $p$, then [5, Lemma 5.3] shows that $C$ contains a normal subgroup $H$ such that $C / H$ is an infinite elementary abelian $p$-group, and then [5, Lemma 2.3] implies that $C$ is a BFC-group. Thus we may assume that $A / A^{\prime} A^{p}$ is finite for all primes $p$. Since $G / A^{\prime}$ satisfies max-n, by [11, Theorem 5.34], the torsion subgroup $P / A^{\prime}$ of $A / A^{\prime}$ is bounded and hence $A / A^{\prime}=P / A^{\prime} \times E / A^{\prime}$ for some torsion-free subgroup $E / A^{\prime}$. Since $A / A^{\prime} A^{p}$ is finite $P / A^{\prime}$ is also finite. Hence $P$, the torsion subgroup of $A$, is finite and since $C / A$ is polycyclic-by-finite the torsion subgroup of $C$ is also finite. Since $C$ is a locally FC-group, [5, Lemma 2.1] gives the result.

Theorem 3.2. Let $G$ be a finitely generated soluble-by-finite group satisfying max(non-BFC). Suppose that $K$ is a BFC-group. Then $G$ has a series of normal subgroups $F \leqslant A \leqslant G$ satisfying the following conditions:

(i) $F$ is finite;

(ii) $A / F$ is abelian; 
(iii) $G / A$ is finitely generated, abelian-by-finite and torsion-free; and

(iv) $A / F$ is a finitely generated $\mathbb{Z}\langle g\rangle$-module for each element $g \in G \backslash A$.

Furthermore, if $K / K^{\prime} K^{p}$ is finite for each prime $p$, then $F$ may be chosen such that $A / F$ is torsion-free and minimax. In this case $G$ is also minimax.

Proof. Let

$$
\mathcal{S}=\left\{H \triangleleft G \mid K \leqslant H \text { and } H^{\prime} \text { is finite }\right\} .
$$

Then $K \in \mathcal{S}$ and $\mathcal{S}$ has a maximal element $A$, since $G / K$ is polycyclic-by-finite. Then $A$ is a BFC-group and $A$ is not finitely generated otherwise $A$ has the maximal condition, whence $K$ is also finitely generated, a contradiction.

We show first that $G / A$ is torsion-free. To this end let $U / A$ be a finite subgroup of $G / A$. Then $U$ is not finitely generated so $U$ is a locally FC-group and by [5, Lemma 2.1] its torsion subgroup is periodic. Since $G / K^{\prime}$ satisfies max-n, by [11, Theorem 5.34] the torsion subgroup $P / K^{\prime}$ of $K / K^{\prime}$ is bounded and hence $K / K^{\prime}=P / K^{\prime} \times E / K^{\prime}$, for some torsion-free subgroup $E / K^{\prime}$.

If $K / K^{\prime} K^{p}$ is finite for all primes $p$, then $P$ is finite and since $U / K$ is polycyclicby-finite the torsion subgroup of $U$ is also finite, and hence $U$ is a BFC-group by [5, Lemma 2.1]. If $K / K^{\prime} K^{p}$ is infinite for some prime $p$, then $A / A^{\prime} A^{p}$ is also infinite, since $A / K A^{\prime}$ is a finitely generated abelian group and [5, Lemma 3.2] implies that $U$ contains a normal subgroup $W$ such that $U / W$ is an infinite elementary abelian $p$-group. Hence $U$ is a BFC-group in this case also, by [5, Lemma 2.3].

Now let $F$ be a finite $G$-invariant subgroup of $A$ such that $A / F$ is abelian. Let $U / A=\left\{u_{1} A, \ldots, u_{m} A\right\}$ and write $V / F=\left\langle u_{1} F, \ldots, u_{m} F\right\rangle^{U / F}$. Since $U / F$ is a BFCgroup, $V / F$ contains a finite normal subgroup $X / F$ such that $V / X$ is finitely generated and torsion-free abelian. By a theorem of Jategoankar and Roseblade (see, for example, [9, Theorem 12.3.13]), $G / F$ is residually finite. Therefore, $G / F$ contains a normal subgroup $D / F$ of finite index such that $D / F \cap X / F=1$. Let $D_{1}=D \cap A$. Since $D_{1} / F$ and $V / F$ are normal in $U / F$ we have $\left[V / F, D_{1} / F\right] \leqslant V / F \cap D_{1} / F=Y / F$, say. By the choice of $D_{1}, Y / F$ is torsion-free. On the other hand, $\left[V / F, D_{1} / F\right] \leqslant(U / F)^{\prime}$ and $(U / F)^{\prime}$ is finite. Therefore, $\left[V / F, D_{1} / F\right] \leqslant Y / F \cap(U / F)^{\prime}=1$ and hence $V / F \leqslant C_{G / F}\left(D_{1} / F\right)$. Since $V A=U$ it follows that $A / F \supsetneqq C_{G / F}\left(D_{1} / F\right)$, contrary to Lemma 3.1. Thus $G / A$ is torsion-free.

Next, let $g \in G \backslash A$, let $Q=\langle A, g\rangle$ and suppose that $Q$ is a BFC-group. If $\langle z\rangle=C_{\langle g\rangle}\left(Q^{\prime}\right)$, then $|\langle g\rangle:\langle z\rangle|$ is finite. For each element $a \in A$ we have $a^{z}=a b$ for some element $b \in Q^{\prime}$. If $r=\left|Q^{\prime}\right|$, then $z^{-r} a z^{r}=a b^{r}=a$ and hence $z^{r} \in C_{G}(A)$, so that $z^{r} \in A$, by Lemma 3.1. Thus $g \in A$ since $G / A$ is torsion-free. This contradiction shows that $\langle A / F, g F\rangle^{\prime}$ is infinite. By Hall's Theorem [11, Theorem 5.34], $G / F$ satisfies max-n and hence the torsion subgroup of $A / F$ is bounded. Lemma 2.4 now shows that $A / F$ is a finitely generated $\mathbb{Z}\langle g\rangle$-module.

If $K / K^{\prime} K^{p}$ is finite for all primes $p$, then, as we noted above, the torsion subgroup of $A$ is finite and we let $F$ be this subgroup in this case. Since $G / F$ satisfies max-n, 
$A / F$ is a finitely generated $\mathbb{Z}(G / A)$-module. By a theorem of Hall (see $[\mathbf{1 1}$, Corollary 1Lemma 9.53]) $A / F$ contains a free abelian subgroup $M / F$ such that $A / M$ is periodic and $\pi(A / M)$ is finite. Since $A / A^{\prime} A^{p}$ is finite for each prime $p$, it follows that $M / F$ is finitely generated and hence $A / F$ is minimax in this case. If $K / K^{\prime} K^{p}$ is infinite for some prime $p$, then we let $F=A^{\prime}$.

The group $A$ has a finite series of $G$-invariant subgroups

$$
F=A_{0} \leqslant A_{1} \leqslant \cdots \leqslant A_{k}=A
$$

such that $A_{i+1} / A_{i}$ is elementary abelian for $0 \leqslant i \leqslant k-2$, and $A_{k} / A_{k-1}$ is torsionfree. The group ring $\mathbb{Z}\langle g\rangle$ is Noetherian so $A / F$ is a Noetherian $\mathbb{Z}\langle g\rangle$-module and hence $A_{i+1} / A_{i}$ is a finitely generated $\mathbb{Z}\langle g\rangle$-module for each $i$. Suppose first that $A_{k} / A_{k-1}$ is not of finite rank. Then, for each $g \in G \backslash C_{G}\left(A_{i+1} / A_{i}\right)$, [6, Lemma 18 and its Corollary] show that $G / C_{G}\left(A_{i+1} / A_{i}\right)$ is abelian-by-finite for $0 \leqslant i \leqslant k-1$. If $A_{k} / A_{k-1}$ is of finite rank, then there is a series of $G$-invariant subgroups $A_{k-1}=B_{0} \leqslant B_{1} \leqslant B_{l}=$ $A_{k}$ with $B_{i+1} / B_{i} G$-rationally irreducible, for $i=0, \ldots, l-1$. By Clifford's Theorem and Mal'tsev's Theorem [13, Lemma 3.5], $S / C_{S}\left(B_{i+1} / B_{i}\right)$ is abelian-by-finite and hence $G / C_{G}\left(B_{i+1} / B_{i}\right)$ is also abelian-by-finite.

In either case, if

$$
L=C_{G}\left(A_{1} / A_{0}\right) \cap \cdots \cap C_{G}\left(A_{k-1} / A_{k-2}\right) \cap C_{G}\left(B_{1} / B_{0}\right) \cap \cdots C_{G}\left(B_{l} / B_{l-1}\right)
$$

(setting $B_{0}=A_{k-1}$ and $B_{1}=A_{k}$ if $A_{k} / A_{k-1}$ is not of finite rank), we have that $G / L$ is abelian-by-finite. If $g \in L \backslash A$, then $\langle A / F, g F\rangle^{\prime}$ is infinite, $\langle A / F, g F\rangle$ is nilpotent and also finitely generated, which is a contradiction. Hence $L \leqslant A$ so $A=L$, and hence $G / A$ is abelian-by-finite. This completes the proof.

Let $p$ be a prime. In $[8]$ it is shown that if $\langle x\rangle$ is an infinite cyclic group and if $K=\mathbb{F}_{p}\langle x\rangle$ is the group ring over the field with $p$ elements, then the group $G=K_{+} \rtimes\langle x\rangle$, where $K_{+}$is the additive group of $K$, satisfies the maximal condition on non-abelian subgroups. Hence $G$ also satisfies max-(non-BFC) and it is finitely generated. The necessary conditions obtained in Theorem 3.2 are also sufficient as we now show.

Lemma 3.3. Let $G$ be a group having a series of normal subgroups $F \leqslant A \leqslant G$ satisfying conditions (i)-(iv) of Theorem 3.2. Then $G$ satisfies max-(non-BFC).

Proof. Let $H_{1} \leqslant H_{2} \leqslant \cdots \leqslant H_{n} \leqslant \cdots$ be an ascending chain of subgroups, each having infinite derived subgroup. Since $A$ is a BFC-group, $H_{n} \not A$ for all $n \in \mathbb{N}$. If $g \in H_{1} \backslash A$, then $A / F$ is a finitely generated $\mathbb{Z}\langle g\rangle$-module and $\mathbb{Z}\langle g\rangle$ is a Noetherian group ring. Thus $A / F$ is a Noetherian $\mathbb{Z}\langle g\rangle$-module and, as $\left(A \cap H_{i}\right) F / F$ is a $\mathbb{Z}\langle g\rangle$-submodule of $A / F$, there exists $r \in \mathbb{N}$ such that $\left(A \cap H_{r}\right) F=\left(A \cap H_{n}\right) F$ for all $n \geqslant r$. Since $F$ is finite there exists $s \in \mathbb{N}$ such that $A \cap H_{s}=A \cap H_{n}$ for all $n \geqslant s$ and since $G / A$ satisfies the maximum condition, there exists $t \in \mathbb{N}$ such that $H_{t} A=H_{n} A$ for all $n \geqslant t$. Thus if $k=\max (s, t), H_{n}=H_{k}$ for all $n \geqslant k$, so the result follows.

Next we handle the case when $K$ is not a BFC-group. 
Theorem 3.4. Let $G$ be a finitely generated soluble-by-finite group satisfying max(non-BFC). Suppose that $K^{\prime}$ is infinite. Then $G$ has a series of normal subgroups $F \leqslant$ $T \leqslant A \leqslant G$ satisfying the following conditions:

(i) $F$ is finite;

(ii) $T=F D$, where $D$ is a divisible Cernikov $q$-subgroup, for some prime $q$ and $D \leqslant Z(A)$

(iii) $A / T$ is abelian and torsion-free;

(iv) $G / A$ is finitely generated, abelian-by-finite and torsion-free;

(v) $A / T$ is a finitely generated $\mathbb{Z}\langle g\rangle$-module for each $g \in G \backslash A$; and

(vi) if the subgroup $H$ has infinite derived subgroup, then $D \leqslant H$.

Proof. Clearly, $K$ is a locally FC-group and by [5, Lemma 2.3] $K / K^{\prime} K^{p}$ is finite for all primes $p$. Let $P$ be the torsion subgroup of $K$. Then $K^{\prime} \leqslant P$ and $K / P$ is minimax by [5, Lemma 6.1]. Thus $G / P$ is also minimax since $G / K$ is polycyclic-by-finite. Let

$$
\mathcal{S}=\left\{H \triangleleft G \mid K \leqslant H \text { and }(H / P)^{\prime} \text { is finite }\right\} .
$$

Then $K \in \mathcal{S}$ and since $G / K$ satisfies the maximum condition, $\mathcal{S}$ has a maximal element $A$. Let $T / P$ be the torsion subgroup of $A / P$. Since $A / K$ is polycylic-by-finite and $K / P$ is torsion-free, the torsion subgroup $T / P$ of $A / P$ is finite. By Hall's Theorem [11, Theorem 5.34] $G / K^{\prime}$ satisfies max-n so $P / K^{\prime}$ is bounded and hence finite since $K / K^{\prime} K^{p}$ is finite for each prime $p$. If $A$ is finitely generated, then $A / P$ has the maximum condition, whence $K / K^{\prime}$ is finitely generated, contrary to the choice of $K$. Hence $A$ is a locally FC-group and $A / T$ is a torsion-free abelian group by [5, Lemma 2.1].

Let $C / T=C_{G / T}(A / T)$. Since $A / T$ is not finitely generated then neither is $C / T$, as in the proof of Lemma 3.1. Hence $C / P$ is a locally FC-group and as in Lemma 3.1 we have $C=A$. If $U / A$ is a finite subgroup of $G / A$, then $U / T$ is not finitely generated so $U / T$ is a locally FC-group. Thus $(U / T)^{\prime}$ is periodic and since $A / T$ is torsion-free we have $U / T \leqslant C_{G / T}(A / T)=A / T$. Thus $G / A$ is torsion-free.

Let $g \in G \backslash A$. If $\langle A, g\rangle / T$ is a BFC-group, then, as above, $g T \in C_{G / T}(A / T)=A / T$, a contradiction. Hence $\langle A, g\rangle / T$ has infinite derived subgroup and Lemma 2.4 implies that $A / T$ is a finitely generated $\mathbb{Z}\langle g\rangle$-module.

Since $A$ is a locally FC-group, [5, Lemma 6.5] implies that $A^{\mathfrak{F}} \leqslant T$ so that $A / A^{\mathfrak{F}}$ is not finitely generated. By [5, Theorem 6.8] $A$ contains a finite $A$-invariant subgroup $F_{1}$ and a divisible Cernikov $q$-subgroup $D$, for some prime $q$ such that $T=D F_{1}$ and $D \leqslant Z(A)$. If $X$ is a finitely generated subgroup of $G$ such that $G=A X$, then $F=F_{1}^{X} \leqslant T$ and it is easy to see that $F$ is finite. Of course, $F$ is $G$-invariant.

Finally, let $H$ be a subgroup such that $H^{\prime}$ is infinite. Suppose that $E=D \cap H \neq D$. Then $D / E$ is a non-trivial divisible Cernikov group and by [5, Lemma 2.6] it has the maximal condition on $H$-invariant subgroups. This is a contradiction and the result follows. 
Several additional facts can be obtained concerning groups in this case and we note these here.

Lemma 3.5. Let $G$ be a finitely generated soluble-by-finite group satisfying max(non-BFC). Suppose that $K^{\prime}$ is infinite. Using the notation of Theorem 3.4 the following hold:

(i) $A$ contains a subgroup $B \geqslant T$ such that $B / T$ is finitely generated and $A / B$ is a Prüfer q-group for some prime $q$;

(ii) $G$ is minimax;

(iii) $A^{\prime}$ is infinite; and

(iv) if $g \notin C_{G}(D)$, then $g$ induces an infinitely irreducible automorphism on $D$.

Proof. Item (i) follows from Theorem 6.8 of [5] and (ii) is then immediate. Item (iii) is clear from the definition of $A$. To prove item (iv) let $g \notin C_{G}(D)$. If $\langle D, g\rangle$ is a BFC-group, then $D \leqslant Z(\langle D, g\rangle)$ by [12, Theorem 1.9], a contradiction. Thus $\langle D, g\rangle^{\prime}$ is infinite. Now $D$ has a finite series of $\langle g\rangle$-invariant divisible subgroups $1=D_{0} \leqslant D_{1} \leqslant \cdots \leqslant D_{t}=D$, every factor of which is $\langle g\rangle$-infinitely irreducible. In particular, either $\left[D_{i+1}, g\right] \leqslant D_{i}$ or $\left[D_{i+1} / D_{i}, g\right]=D_{i+1} / D_{i}$ for $0 \leqslant i \leqslant t-1$. Since $\langle D, g\rangle^{\prime}$ is infinite there is a least integer $m$ such that $\left[D_{m+1} / D_{m}, g\right]=D_{m+1} / D_{m}$ and it follows that $\left\langle D_{m+1} / D_{m}, g D_{m}\right\rangle^{\prime}$ is infinite. Clearly, $D=D_{m+1}$, by [5, Lemma 2.6]. If $D_{m} \neq 1$, then $\left[D_{m}, g\right]=1$, again using [5, Lemma 2.6]. By [4, Lemma 4.14] $D$ contains a $\langle g\rangle$-invariant divisible subgroup $M$, which is $\langle g\rangle$-infinitely irreducible such that $D_{m} M=D,[M, g]=M$ and $M \cap D_{m}$ is finite. As above, $D=M$, and hence $g$ acts on $D$ infinitely irreducibly.

Finally, we show that the necessary conditions of Theorem 3.4 are also sufficient.

Lemma 3.6. Let the group $G$ satisfy the conditions of Theorem 3.4. Then $G$ satisfies $\max -($ non- $B F C)$.

Proof. Let $H_{1} \leqslant H_{2} \leqslant \cdots \leqslant H_{n} \leqslant \cdots$ be an ascending series of subgroups such that $H_{n}^{\prime}$ is infinite for each $n \in \mathbb{N}$. Since $D \leqslant H_{n}$ for each $n \in \mathbb{N}$ the result follows by Lemma 3.3 applied to $G / D$.

\section{References}

1. B. Bruno and R. E. Phillips, On minimal conditions related to Miller-Moreno type groups, Rend. Sem. Mat. Univ. Padova 69 (1983), 153-168.

2. S. N. ČERnikOv, Groups with a minimality condition for nonabelian subgroups, in Groups with restricted subgroups, pp. 96-106 (Naukova Dumka, Kiev, 1971).

3. M. R. Dixon and L. A. Kurdachenko, Groups with the maximum condition on nonnilpotent subgroups, J. Group Theory 4 (2001), 75-87.

4. M. R. Dixon and L. A. Kurdachenko, Locally nilpotent groups with the maximum condition on non-nilpotent subgroups, Glasgow Math. J. 43 (2001), 85-102. 
5. M. R. Dixon and L. A. Kurdachenko, Groups with the maximal condition on nonBFC subgroups, Algebra Colloq., in press.

6. H. HeineKen and L. A. Kurdachenko, Groups with subnormality for all subgroups that are not finitely generated, Annli Mat. Pura Appl. 169 (1995), 203-232.

7. L. A. Kurdachenko and H. Smith, Groups with the maximal condition on nonsubnormal subgroups, Boll. UMI B 10 (1996), 441-460.

8. L. A. Kurdachenko And D. I. Zaicev, Groups with the maximum condition for nonabelian subgroups, Ukrain. Mat. Zh. 43 (1991), 925-930. (English translation: Ukraine Math. J. 43 (1991), 863-868.)

9. D. PASSMAn, The algebraic structure of group rings, Pure and Applied Mathematics Series (Wiley, 1977).

10. R. E. Phillips and J. S. Wilson, On certain minimal conditions for infinite groups, $J$. Alg. 51 (1978), 41-68.

11. D. J. S. Robinson, Finiteness conditions and generalized soluble groups, vols 1 and 2, Ergebnisse der Mathematik und ihrer Grenzgebiete, vols 62 and 63 (Springer, 1972).

12. M. J. Tomkinson, FC-groups (Pitman, 1984).

13. B. A. F. Wehrfritz, Infinite linear groups, Ergebnisse der Mathematik und ihrer Grenzgebiete, vol. 76 (Springer, 1973). 